\title{
Light Scalars and the Generation of Density Perturbations During Preheating or Inflaton Decay
}

\author{
Lotty Ackerman, ${ }^{1}$ Christian W. Bauer, ${ }^{1}$ Michael L. Graesser, ${ }^{1}$ and Mark B. Wise ${ }^{1}$ \\ ${ }^{1}$ California Institute of Technology, Pasadena, CA 91125
}

\begin{abstract}
Reheating after inflation can occur through inflaton decay or efficient parametric resonant production of particles from the oscillation of the inflaton. If the particles produced interact with scalars that were light during inflation, then significant super-horizon density perturbations are generated during this era. These perturbations can be highly non-Gaussian.
\end{abstract}

Measurements of the cosmic microwave background radiation [1, 2] have clearly shown the presence of superhorizon primordial density fluctuations at roughly one part in $10^{5}$. Inflation provides a natural explanation for such density fluctuations, since vacuum fluctuations of the inflaton (or any other light scalar field) get pushed outside of the horizon and enter at a much later time as classical density perturbations [3]. Recently, Dvali, Gruzinov and Zaldarriaga [4, 5] and Kofman [6] (DGZK) have shown in a number of scenarios how the interactions of such additional light fields to, e.g. the inflaton, could also generate adiabatic density fluctuations, independent of those created by the inflaton dynamics. In this scenario the size of non-Gaussian perturbations can be much larger than what occurs in single-field inflationary models [5, 7].

This is achieved by coupling a light scalar field to a heavier field that at some time subsequent to inflation dominates the energy of the Universe, such that the particle properties of this heavier field are modified by the fluctuations of the light field. When the heavier particle decays, spatial fluctuations in either its mass or its decay width generate energy density perturbations in the radiation. This is because before reheating the universe is matter dominated, with the oscillating heavier particle dominating the total energy density, while after the decay the universe is radiation dominated. As the energy density in matter redshifts slower than energy density in radiation, regions of the universe where the decay occurs at a later time stay matter dominated longer and will be denser than regions where decay happens earlier. This gives density perturbations of order

$$
\frac{\delta \rho}{\rho} \sim-\frac{\delta \Gamma}{\Gamma} \sim \frac{\delta \tau}{\tau}
$$

where

$$
\tau \equiv t_{\mathrm{RH}}-t_{0}
$$

is the time between the end of inflation $\left(t_{0}\right)$ and reheating $\left(t_{\mathrm{RH}}\right)$. The evolution of density perturbations in this scenario has been studied in detail in [4, 8].

In a similar way, modifications to the particle properties of the particles produced during reheating can also introduce energy density perturbations. Density perturbations are created if the decay products interact with fields that were light during inflation.
To see this, we need to discuss how the inflaton reheats. Suppose reheating occurs through direct (Born) decay of the inflaton. Then a fluctuation in the mass of the decay product $\chi$ modifies the inflaton decay width, because of the dependence of the available phase space on the masses of the final state particles. These lead to calculable density perturbations since the exact dependence of the width on the mass of the light particles can be computed in any given model $[7,9]$. If, for example, the inflaton decays via $\Phi \rightarrow \chi \chi$ then the tree-level decay width is modified from phase space by an amount

$$
\frac{\delta \Gamma}{\Gamma}=-2 \frac{\delta m_{\chi}^{2}}{m_{\phi}^{2}-4 m_{\chi}^{2}}+2\left(\frac{\delta m_{\chi}^{2}}{m_{\phi}^{2}-4 m_{\chi}^{2}}\right)^{2}+\cdots .
$$

If $\Phi$ decays near threshold, then the resulting density perturbation dependence on $\delta m_{\chi}^{2}$ can be large and highly non-linear.

We expect a $\delta m_{\chi}^{2}$ with a super-horizon spatial variation to be generated if $\chi$ interacts with a field $\sigma$ that was light during the inflationary era and through to the era of reheating. Note that even in the absence of direct couplings of the fields $\chi$ and $\sigma$, they are expected to interact indirectly through some intermediate states. Quantum corrections will typically generate a dependence of $m_{\chi}^{2}$ on the super-horizon fluctuations of $\sigma$ at some order in perturbation theory, as indicated by Fig. (1) In this paper we focus mainly on the effect that fluctuations in the mass of the particles produced during reheating or preheating have on density perturbations.

Besides reheating through direct Born decay, the inflaton may instead reheat the universe through parametric resonance (preheating) 10, 11, 12, 13, 14]. Preheating can be very efficient and be completed very soon after inflation, within $\mathcal{O}(10-100)$ oscillations of the inflaton field about its minimum. Whether this process of reheating dominates over the Born decay into bosons or fermions depends on the parameters of the model ${ }^{1}$.

In the scenario of DGZK, additional density perturbations can be created during preheating, by modifying the time it takes for parametric resonance to complete

\footnotetext{
1 The growth of perturbations during the matter-dominated era of the oscillating inflaton has been studied in 15$]$ and, if parametric resonance occurs, in [16].
} 


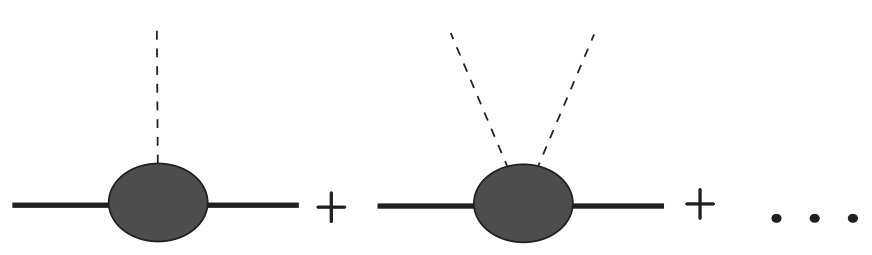

FIG. 1: Quantum corrections may generate a dependence of $m_{\chi}^{2}$ on super-horizon fluctuations in $\sigma$.

and for the universe to thermalize. The size of this time interval depends on the parameters of the model, and in particular on the mass of the produced particles, which we discuss below in a simple model. This is the main subject of this paper. Depending on how efficient preheating is, the size of the time interval can have a weak or strong sensitivity to the mass of the decay products.

We use the canonical model of preheating and add a scalar $\sigma$ which we assume is light during inflation so that it acquires super-horizon perturbations $\delta \sigma(x) \sim H_{\mathrm{inf}}$ during that era. For this to occur it is necessary that during inflation its mass satisfies $m_{\sigma}<H_{\text {inf. }} \sigma$ is assumed to interact more strongly with the $\chi$ compared to $\Phi$. The interactions we consider are

$$
-\mathcal{L}_{I}=\frac{g^{2}}{2} \Phi^{2} \chi^{2}+\mu \chi^{2} \sigma+\frac{\lambda}{2} \chi^{2} \sigma^{2}+\frac{m_{\chi}^{2}}{2} \chi^{2}+\frac{m_{\sigma}^{2}}{2} \sigma^{2}
$$

A $Z_{2}$ symmetry $\chi \rightarrow-\chi$ has been imposed for simplicity. Self-interactions $\sigma^{4}$ and $\chi^{4}$ are assumed to be irrelevant during the first stage of preheating defined below. We assume that at the end of inflation the fields $\chi$ and $\sigma$ are near enough to the minimum of their potential so that we can neglect the motion of their zero modes.

Inflation ends when $t=t_{0} \simeq 1 / m_{\Phi}$ and is followed by a matter-dominated era described by rapid oscillations of the inflaton about the minimum of its potential which we assume to be

$$
V(\Phi)=\frac{1}{2} m_{\Phi}^{2} \Phi^{2}
$$

For simplicity we assume that the inflationary potential is also described by this simple quadratic form, giving rise to chaotic inflation [17]. During inflation, $H_{\mathrm{inf}} \simeq m_{\Phi}$. At the end of inflation $\Phi=\Phi_{0} \simeq m_{\mathrm{pl}} / 3$ and thereafter decays as $\Phi(t) \simeq m_{\mathrm{pl}} / 3 m_{\Phi} t$.

For large enough coupling $g$, these oscillations trigger parametric resonance, and the energy density in $\chi$ increases exponentially [10]. If this process is efficient, the universe eventually is dominated by the $\chi$ particles, which then thermalize the universe at some later time through its interactions with Standard Model or Grand Unified Model particles.

The perturbations in the inflaton give rise to adiabatic density perturbations, whose size depend on the form of the inflaton potential. In this letter we concentrate on the density perturbations generated from the fluctuations in the $\sigma$ scalar field. In de Sitter space 18

$$
\begin{gathered}
\left\langle\sigma^{2}(0)\right\rangle=\frac{H_{\mathrm{inf}}^{2}}{4 \pi^{2}} N, \\
\langle\sigma(x) \sigma(y)\rangle=\frac{H_{\mathrm{inf}}^{2}}{4 \pi^{2}},
\end{gathered}
$$

where in Eq. (6) $N$ is the number of e-foldings during inflation. In Eq. (7) the comoving coordinates $x, y$ are well seperated and we neglect the logarithmic dependence on $|x-y|$.

The $\chi$ field does not acquire super-horizon perturbations because its effective mass

$$
m_{\chi, \mathrm{eff}}^{2}=m_{\chi}^{2}+g^{2}|\Phi|^{2}+\lambda \frac{H_{\mathrm{inf}}^{2}}{4 \pi^{2}} N
$$

during inflation is larger than the Hubble parameter for parameter values which allow for efficient parametric resonance. Henceforth we absorb the $\lambda H_{\text {inf }}^{2} N /\left(4 \pi^{2}\right)$ into $m_{\chi}^{2}$. Treating $\sigma$ as an external field, its fluctuations can be absorbed into fluctuations in the mass of the field $\chi$,

$$
\delta m_{\chi}^{2}=2 \mu \delta \sigma+\lambda \delta \sigma^{2}
$$

where we have used

$$
\delta \sigma \equiv \sigma-\langle\sigma\rangle, \quad \delta \sigma^{2} \equiv \sigma^{2}-\left\langle\sigma^{2}\right\rangle
$$

and we will impose $\langle\sigma\rangle=0$. The size of the fluctuations $\delta m_{\chi}^{2}$ is determined by the two-point function

$$
\left\langle\delta m_{\chi}^{2}(x) \delta m_{\chi}^{2}(y)\right\rangle=4 \mu^{2}\langle\sigma(x) \sigma(y)\rangle+2 \lambda^{2}\langle\sigma(x) \sigma(y)\rangle^{2}
$$

Using Eqs. (6) and (7) we find the fluctuations for widely separated comoving coordinates $x$ and $y$ to be of order

$$
\delta m_{\chi}^{2} \sim \sqrt{\mu^{2} H_{\mathrm{inf}}^{2}+\lambda^{2} H_{\mathrm{inf}}^{4}} .
$$

While the field $\sigma(x)$ is Gaussian, the fluctuation $\delta m_{\chi}^{2}$ is only Gaussian for $\lambda=0$. For $\lambda H_{\text {inf }}^{2} \gg \mu H_{\text {inf }}, \delta m_{\chi}^{2}$ is highly non-Gaussian. For example, consider in this limit the three-point function for equally separated comoving coordinates. One finds for the analog of skewness

$$
\begin{aligned}
& \frac{\left\langle\delta m_{\chi}^{2}(x) \delta m_{\chi}^{2}(y) \delta m_{\chi}^{2}(z)\right\rangle}{\left\langle\delta m_{\chi}^{2}(x) \delta m_{\chi}^{2}(y)\right\rangle^{3 / 2}} \\
& \quad=\frac{8\langle\sigma(x) \sigma(y)\rangle\langle\sigma(y) \sigma(z)\rangle\langle\sigma(z) \sigma(x)\rangle}{2^{3 / 2}\langle\sigma(x) \sigma(y)\rangle^{3}}=2 \sqrt{2}
\end{aligned}
$$

For the remainder of this paper we set $\mu=0$ which corresponds to imposing a $\sigma \rightarrow-\sigma$ symmetry. We make this decision to simplify the analysis of the backreaction of $\chi$ on $\sigma$ discussed below. Then

$$
\frac{\delta m_{\chi}^{2}}{m_{\Phi}^{2}} \sim \lambda \frac{H_{\mathrm{inf}}^{2}}{m_{\Phi}^{2}} \simeq \lambda
$$

These fluctuations in $\delta m_{\chi}^{2}$ are non-Gaussian and always positive. 
This situation would be excluded if this were the only source of density perturbations. A more interesting scenario in this situation would be if the dominant source of perturbations came from the inflaton potential. Then the perturbations generated during preheating providing a sub-dominant, non-Gaussian contribution. Since here the source for the non-Gaussian perturbations is not the same as the source -the inflaton- providing the dominant Gaussian contribution, the current limits on nonGaussianity [19] do not apply, since those limits assume that the non-Gaussian and Gaussian perturbations are generated by the same field.

We define preheating to last until significant particle production of $\chi$ occurs and the energy densities in $\Phi$ and $\chi$ become equal. The duration of this stage depends on $m_{\chi}$ and coupling constant $g$,

$$
\tau=\tau\left(g, m_{\chi}\right) .
$$

Fluctuations in $m_{\chi}$ and the coupling $g$ give rise to density fluctuations from Eq. (1).

Fluctuations in $g$ can be generated if it is replaced by an effective coupling

$$
g_{\mathrm{eff}}^{2}=g^{2}\left(1+\frac{\sigma^{2}}{M^{2}}\right),
$$

where $M$ is some mass scale 20]. The $\sigma$ dependence of $g_{\text {eff }}$ generates non-Gaussian perturbations $\delta_{g} \equiv \delta g^{2} / g^{2}=$ $H_{\text {inf }}^{2} / M^{2}$. It also modifies the large time-dependent mass of $\chi$, an effect that is distinct from modifying $m_{\chi}$. Here too we have to worry about the backreaction of $\chi$ on $\sigma$.

Next we describe our numerical method for determining the energy density in $\chi$ during preheating. Neglecting the backreaction of $\chi$ on the inflaton, which only becomes significant at the end of the preheating stage when $\rho_{\chi}=\rho_{\Phi}$ [12], the equation of motion for the fields $\chi \equiv \hat{\chi}\left(a_{0} / a\right)^{3 / 2}$ ( $a$ is the scale factor) and $\sigma$ are

$$
\begin{gathered}
\hat{\chi}_{k}^{\prime \prime}+\left[A_{k}+2 q \cos \left(2\left(z-z_{0}\right)\right)\right] \hat{\chi}_{k}=0 \\
\delta \sigma^{\prime \prime}+\frac{2}{z} \delta \sigma^{\prime}+m_{\sigma, \mathrm{eff}}^{2} \delta \sigma=0,
\end{gathered}
$$

where derivatives are with respect to $z \equiv m_{\Phi} t$ and we have chosen $z_{0} \equiv 1$. We have defined

$$
\begin{aligned}
q & =\frac{g^{2} \Phi_{0}^{2} a_{0}^{3}}{4 a^{3} m_{\Phi}^{2}} \equiv q_{0} \frac{a_{0}^{3}}{a^{3}}, \quad a_{0} \equiv a\left(t_{0}\right) \\
A_{k} & =\frac{1}{m_{\Phi}^{2}}\left(k^{2} \frac{a_{0}^{2}}{a^{2}}+\widetilde{m}_{\chi}^{2}\right)+2 q,
\end{aligned}
$$

and the mass parameters are given by

$$
\begin{aligned}
\widetilde{m}_{\chi}^{2} & =m_{\chi}^{2}+\lambda \delta \sigma^{2} \\
m_{\sigma, \text { eff }}^{2} & =m_{\sigma}^{2}+\lambda \chi^{2} .
\end{aligned}
$$

The equation for $\hat{\chi}_{k}$ describes a time-dependent harmonic oscillator with frequency $\Omega_{k}^{2}=$
$m_{\Phi}^{2}\left[A_{k}+2 q \cos \left(2\left(z-z_{0}\right)\right)\right]$. In the limit of a static universe and constant $\delta \sigma$ this equation reduces to the Mathieu equation.

Efficient parametric resonance requires $q_{0} \gg 1$ and $\widetilde{m}_{\chi} \lesssim m_{\phi}$. Note that we included a term of order $\lambda H_{\text {inf }}^{2} N$ into the definition of $m_{\chi}^{2}$, where $N$ is the number of efoldings during inflation. The bound $\widetilde{m}_{\chi} \lesssim m_{\phi}$ therefore implies $N \lesssim \lambda^{-1}$. For the values of $\lambda$ we consider, this is a very weak bound on the number of e-foldings during inflation.

For a given value of $k$ the energy density in $\chi$ is

$$
\rho_{k}(z)=\Omega_{k}(z) N_{k}(z),
$$

where $N_{k}(t)$ is the number density for a mode with given wave number $k$. The number density can be calculated by numerically solving for the Bogolyubov coefficient, giving [1]

$$
N_{k}(t)=\frac{a_{0}^{3}}{2 \Omega_{k} a^{3}(t)}\left(\Omega_{k}^{2}(t)\left|\tilde{\chi}_{k}\right|^{2}+m_{\Phi}^{2}\left|\tilde{\chi}_{k}^{\prime}\right|^{2}\right),
$$

with initial conditions $\tilde{\chi}_{k}\left(t_{0}\right)=1 / \sqrt{2 \Omega_{k}}, m_{\Phi} \tilde{\chi}_{k}^{\prime}\left(t_{0}\right)=$ $-i \sqrt{\Omega_{k} / 2}$. The field $\tilde{\chi}_{k}$ satisfies the same equation as $\hat{\chi}_{k}$ and is related to it (see Appendix B of [11). The energy density is obtained by integrating Eq. (20) to obtain

$$
\rho_{\chi}(z)=\frac{1}{2 \pi^{2}} \int_{0}^{\infty} k^{2} d k \Omega_{k}(z) N_{k}(z) .
$$

The exponentially large number density of $\chi$ particles leads to a large backreaction on $\sigma$ that must be included to correctly determine the size of the effect we are describing. The backreaction of $\chi$ on $\sigma$ can have two effects: first, it can lead to production of large numbers of $\sigma$ particles, and second it gives rise to a large effective mass of the $\sigma$ field. The first effect was analyzed by Felder and Kofman [21] using a numerical lattice simulation of preheating and the subsequent thermalization of the $\chi$ with the $\sigma$ fields. In their Figures 14 and 15 they show the number densities of $\Phi, \chi$ and $\sigma$. Their numerical results show that during preheating the number density in $\sigma$ is much smaller than in either $\chi$ or $\Phi$ and its effect on the evolution of either $n_{\chi}$ or $n_{\Phi}$ is negligible. The second effect is more significant. Once $m_{\sigma \text {,eff }}$ gets larger than $H$, the amplitude $\delta \sigma$ will decrease rapidly [22]. To simplify the analysis we will assume that the dependence of $m_{\sigma, \text { eff }}$ on $m_{\sigma}$ can be neglected. To estimate the time at which the backreaction becomes important, we compare the effective mass $m_{\sigma \text {,eff }}^{2} \sim \lambda\left\langle\chi^{2}\right\rangle$ to the Hubble parameter. The ratio that determines their relative importance can be expressed as

$$
\frac{m_{\sigma, \mathrm{eff}}^{2}}{3 H^{2}}=\frac{2 \lambda}{3 g^{2}} \frac{\rho_{\chi}}{\rho_{\Phi}} \frac{m_{\Phi}^{2}}{H^{2}},
$$

where we have used $m_{\chi, \mathrm{eff}} \simeq g|\Phi|$ and $\rho_{\chi} \simeq g|\Phi| n_{\chi} \simeq$ $g^{2} \Phi^{2}\left\langle\chi^{2}\right\rangle$ [12]. For $\lambda \sim 10^{-5}$ and $H \sim 2 m_{\Phi} / 300$ we find that this backreaction becomes important when $\rho_{\chi} / \rho_{\Phi} \approx$ 
$3 g^{2}$. For $\lambda \sim 10^{-7}$ the backreaction becomes important when $\rho_{\chi} / \rho_{\Phi} \approx 300 g^{2}$. In this letter we will not solve the full coupled set of differential equations, but rather deal with this backreaction by turning off $\delta m_{\chi}^{2}$ at the time $z_{c}$ when $m_{\sigma, \text { eff }}^{2}=3 H^{2}$, i.e. defined implicitly by

$$
\frac{\rho_{\chi}\left(z_{c}\right)}{\rho_{\Phi}\left(z_{c}\right)} \equiv \frac{g^{2}}{\lambda} \frac{2}{3 z_{c}^{2}} .
$$

Although for different values of $\delta m_{\chi}^{2}$ the intercept time $z_{c}$ is different, that difference is second order in $\delta m_{\chi}^{2}$. It is then sufficient to use the $z_{c}$ obtained by setting $\delta m_{\chi}^{2}=0$. If Eq.(24) intercepts $R$ along a plateau corresponding to no particle production, then we make the conservative choice of cutting off the mass fluctuation at the location of the first intercept.

In Fig. 2 we display a logarithmic plot of the ratio $R \equiv \rho_{\chi}(t) / \rho_{\Phi}(t)$ together with Eq. (24) for scenario 1, as defined in Table [ In order to estimate the sensitivity of $z_{\mathrm{RH}}=1+m_{\Phi} \tau$ on $\delta m_{\chi}^{2}$, we show in Fig. [3a magnification of the region where $R\left(z_{\mathrm{RH}}\right)=1$. We also show these plots for the three other scenarios defined in Table \(keeping $m_{\Phi} / m_{\mathrm{pl}}=10^{-6}$ fixed).

\begin{tabular}{c||ccc||c|c} 
scenario & $\mathrm{g}$ & $\lambda$ & $m_{\chi}^{2} / m_{\Phi}^{2}$ & $z_{c}$ & $\kappa_{m}$ \\
\hline 1 & $4 \times 10^{-4}$ & $10^{-7}$ & 0.1 & 88 & 0.8 \\
2 & $6 \times 10^{-4}$ & $10^{-7}$ & 0.4 & 82 & 0.15 \\
3 & $4 \times 10^{-4}$ & $10^{-5}$ & 0.1 & 47 & 0.14 \\
4 & $6 \times 10^{-4}$ & $10^{-5}$ & 0.4 & 50 & 0.06
\end{tabular}

TABLE I: Definition of the four choices parameter sets. Also shown are the numerical results for $z_{c}$ and $\kappa_{m}$, as defined in Eqs (24) and (25).

We are interested in the change in $\tau$ generated by a fluctuation $\delta m_{\chi}^{2} / m_{\Phi}^{2} \simeq \lambda$. Since $\lambda$ is tiny, that change can be expressed as

$$
\frac{\delta \tau}{\tau}=\kappa_{m} \frac{\delta m_{\chi}^{2}}{m_{\Phi}^{2}}
$$

From Table【we can see that the typical $\kappa_{m}$ is $\mathcal{O}(0.1-1)$.

The reader may wonder why we are using larger values for $\delta m_{\chi}^{2}$ that are not consistent with the $\lambda$ we choose. Since the perturbation $\delta \tau$ is linear in $\delta m_{\chi}^{2}$, the $\kappa_{m}$ obtained this way is unchanged if we were to use smaller values for $\delta m_{\chi}^{2}$. The reason for this choice of $\delta m_{\chi}^{2}$ is that the plots are easier to read. We also repeat that $z_{c}$ was determined with the correct $\lambda$.

One may also wonder why the presence of a $\delta m_{\chi}^{2}$ at early times has any effect at all, especially given that it only persists while $R \lesssim 10^{-5}-10^{-3}$. Parametric resonance is dramatic because of stimulated emission. So even if at earlier times the production of $\chi$ particles is affected due to a non-zero $\delta m_{\chi}^{2}$, this will impact the much greater growth occurring at later times. A more detailed numerical simulation, including all the effects of backreaction and scattering, such as done in 21] for preheating

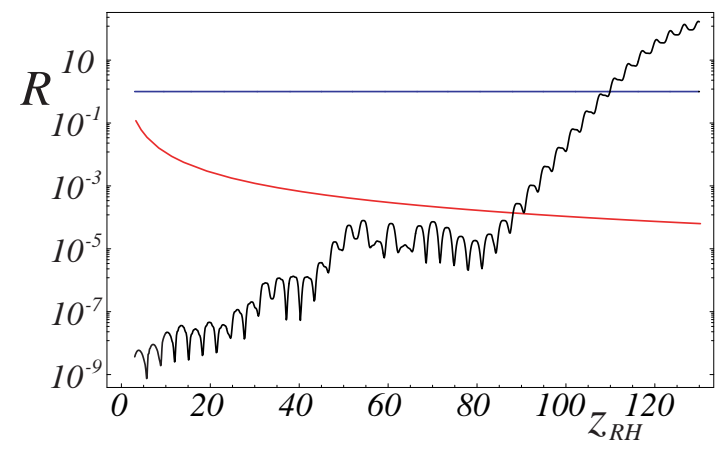

FIG. 2: Logarithmic plot of $R=\rho_{\chi} / \rho_{\Phi}$. The chosen parameters are $g=4 \times 10^{-4}, m_{\Phi}=10^{-6} m_{\mathrm{pl}}, m_{\chi}^{2} / m_{\Phi}^{2}=0.1$. Also shown is Eq. (24) with $\lambda=10^{-7}$.
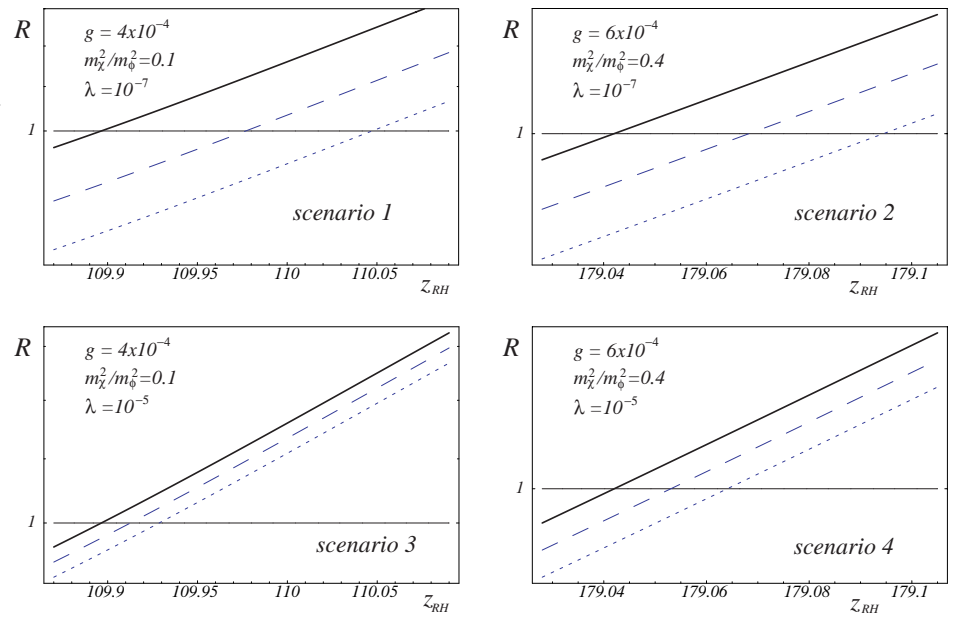

FIG. 3: Logarithmic plot of the effect of the mass fluctuation $\delta m_{\chi}^{2}$ on $z_{\mathrm{RH}}$. The chosen parameters are given in each figure. The solid line corresponds to $\delta m_{\chi}^{2}=0$, while the long and short dashed lines correspond to $\delta m_{\chi}^{2} / m_{\Phi}^{2}=10^{-3}$ and $2 \times$ $10^{-3}$, respectively.
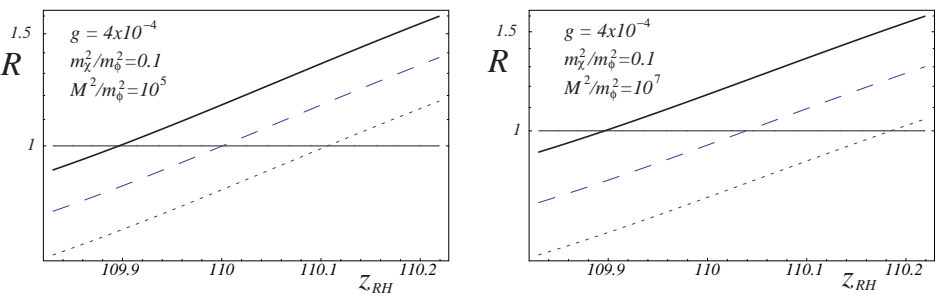

FIG. 4: Logarithmic plot of the effect of the fluctuation in the coupling constant $\delta g^{2}$ on $z_{\mathrm{RH}}$. The chosen parameters are given in each figure. The solid line corresponds to $\delta g^{2}=0$, while the long and short dashed lines correspond to $\delta g^{2} / g^{2}=$ $10^{-3}$ and $2 \times 10^{-3}$, respectively.

without a fluctuating $\sigma$ field, is needed to explore in detail the sensitivity of $\delta \rho / \rho$ to super-horizon fluctuations in $\sigma$.

Mathematically, the intuition expressed above may be expressed in the following way. The density in $\chi$ is ap- 
proximately given by

$$
\rho_{\chi} \simeq \widetilde{N} \frac{a_{0}^{3}}{a^{3}} \exp \left[\int_{t_{0}}^{t_{\mathrm{RH}}} d t \nu(t)\right]
$$

where $\widetilde{N}$ is a prefactor that depends on the parameters of the model. Here $\nu$ is a characteristic exponent leading to exponential growth. We approximate its dependence on $k$ as given by its value near $k \simeq 0$. The coefficient $\nu$ also depends on $m_{\chi}^{2}$, so

$$
\nu=\nu_{0}-\nu_{1} \frac{\delta m_{\chi}^{2}}{m_{\Phi}^{2}} \Theta\left(z_{c}-z\right) .
$$

Numerically we find that $\nu_{1} / \nu_{0} \sim \mathcal{O}(1)$ and is positive. A negative correlation is expected, since both the characteristic exponents of the Mathieu equation and the instability bands are the largest near the kinematic limit $A=2 q$, corresponding to $m_{\chi}=k=0$. Increasing $m_{\chi}^{2}$ removes more instability bands from the available phase space. Using the approximate formula above, we can solve for the change in the reheat time due to a fluctuation $\delta m_{\chi}^{2}$, approximating all the dependence of $\delta \tau$ on $\delta m_{\chi}^{2}$ as occurring from the exponential. This gives

$$
\frac{\delta \tau}{\tau} \simeq-\frac{\nu_{1}}{\nu_{0}} \frac{\delta m_{\chi}^{2}}{m_{\Phi}^{2}} \frac{z_{c}}{z_{\mathrm{RH}}} \simeq \frac{\delta m_{\chi}^{2}}{m_{\Phi}^{2}} \frac{z_{c}}{z_{\mathrm{RH}}} .
$$

This result has $\mathcal{O}(1)$ agreement with our previous numerical computations. (Compare $z_{c} / z_{\mathrm{RH}}$ with $\kappa_{m}$.) It illustrates that $\delta \tau / \tau$ is not suppressed by any very small numbers other than $\delta m_{\chi}^{2} / m_{\Phi}^{2}$.

We also explore the dependence of $\tau$ on fluctuations in $g_{\text {eff }}$ [20]. For non-zero particle number $n_{\chi}$ the interaction (15) introduces a backreaction of $\chi$ on $\sigma$ corresponding to an effective mass $m_{\sigma, \text { eff }}^{2}=g^{2} \Phi^{2}\left\langle\chi^{2}\right\rangle / M^{2}$. As before, we cut off the fluctuation in $g_{\text {eff }}$ when $m_{\sigma, \text { eff }}^{2}=3 H^{2}$. This occurs when

$$
\frac{\rho_{\chi}}{\rho_{\Phi}}=\frac{M^{2}}{m_{\mathrm{pl}}^{2}}
$$

The fluctuation in $g_{\text {eff }}$

$$
\delta_{g} \equiv \frac{\delta g^{2}}{g^{2}}=\frac{H_{\mathrm{inf}}^{2}}{M^{2}},
$$

gives rise to non-Gaussian density perturbations.

In Fig. [4 we display the ratio $R$ for $g=4 \times 10^{-4}$, $m_{\Phi} / m_{\mathrm{pl}}=10^{-6}$ and $m_{\chi}^{2} / m_{\Phi}^{2}=0.1$. We choose two values of $M$ that give $\delta_{g}=10^{-5}$ and $\delta_{g}=10^{-7}$. According to (29), the fluctuation in $g_{\text {eff }}$ is cut off at $\rho_{\chi} / \rho_{\Phi}=10^{-7}$ and $10^{-5}$, respectively, corresponding to $z_{c}=26$ and $z_{c}=47$. For both of these parameters we find that there is a large linear effect which we express as

$$
\frac{\delta \tau}{\tau}=\kappa_{g} \delta_{g}
$$

For $\delta_{g}=10^{-5}$ we find $\kappa_{g}=0.9$ and for $\delta_{g}=10^{-7}$ we find $\kappa_{g}=1.4$. As in the previous case, in obtaining our plots we used larger values of $\delta_{g}$ to determine $\kappa_{g}$.

In conclusion, we have shown that during preheating, interactions of the "decay products" of the inflaton with other light scalar fields can give rise to super-horizon mass fluctuations in these decay products. These fluctuations will then give rise to density perturbations of the universe. Depending on the coupling of the decay products of the inflaton to the light scalar fields, the dominant density perturbations generated from this effect will be either Gaussian or non-Gaussian.

This work was supported by the Department of Energy. under the contract DE-FG03-92ER40701.
[1] G. F. Smoot et al., Astrophys. J. 396, L1 (1992); C. L. Bennett et al., Basic Astrophys. J. 464, L1 (1996).

[2] C. L. Bennett et al., Astrophys. J. Suppl. 148, 1 (2003); D. N. Spergel et al. Astrophys. J. Suppl. 148, 175 (2003).

[3] For a review see e.g. E. W. Kolb and M. S. Turner, The Early Universe, Perseus Publishing, (1990), Cambridge, Massachusetts.

[4] G. Dvali, A. Gruzinov and M. Zaldarriaga, Phys. Rev. D 69, 023505 (2004).

[5] G. Dvali, A. Gruzinov and M. Zaldarriaga, Phys. Rev. D 69, 083505 (2004).

[6] L. Kofman, plenary talk Cosmo03, Chicago, September 2002; astro-ph/0303614

[7] M. Zaldarriaga, Phys. Rev. D 69, 043508 (2004).

[8] S. Tsujikawa, Phys. Rev. D 68, 083510 (2003); A. Mazumdar and M. Postma, Phys. Lett. B 573, 5 (2003), Erratum-ibid. B 585, 295 (2004); F. Vernizzi, Phys. Rev. D 69, 083526 (2004).

[9] M. Postma, JCAP 0403, 006 (2004).
[10] J. H. Traschen and R. H. Brandenberger, Phys. Rev. D 42, 2491 (1990); L. Kofman, A. D. Linde and A. A. Starobinsky, Phys. Rev. Lett. 73, 3195 (1994).

[11] Y. Shtanov, J. H. Traschen and R. H. Brandenberger, Phys. Rev. D 51, 5438 (1995).

[12] L. Kofman, A. Linde and A. Starobinsky, Phys. Rev. D 56, 3258 (1997).

[13] I. Zlatev, G. Huey and P. J. Steinhardt, Phys. Rev. D $\mathbf{5 7}, 2152$ (1998).

[14] R. Micha and I. Tkachev, Phys. Rev. D 70, 043538 (2004).

[15] B. Basset, Phys. Rev. D 56, 3429 (1997); H. Kodama and T. Hamazaki, Prog. Theor. Phys. 96, 949 (1996); T. Hamazaki and H. Kodama, Prog. Theor. Phys. 96, 1123 (1996); Y. Nambu and A. Taruya, Prog. Theor. Phys. 97, 83 (1997); F. Finelli and R. Brandenberger, Phys. Rev. Lett. 82, 1362 (1999).

[16] S. Khlebnikov and I. Tkachev, Phys. Lett. B 390, 80 (1997); S. Khlebnikov and I. Tkachev, Phys. Rev. Lett. 
79, 1607 (1997); S. Khlebnikov and I. Tkachev, Phys. Rev. D 56, 653 (1997); B. Bassett, D. Kaiser and R. Maartens, Phys. Lett. B 455, 84 (1999); B. A. Bassett, F. Tamburini, D. I. Kaiser and R. Maartens, Nucl. Phys. B 561, 188 (1999); F. Finelli and R. Brandenberger, Phys. Rev. D 62, 083502 (2000); F. Finelli and S. Khlebnikov, Phys. Lett. B 504, 309 (2001); F. Finelli and S. Khlebnikov, Phys. Rev. D 65, 043505 (2002); K. Enqvist, A. Jokinen, A. Mazumdar, T. Multamäki, and A. Väihkönen, astro-ph/0411394

[17] A. D. Linde, Phys. Lett. B 108, 389 (1982).
[18] A. D. Linde, Particle Physics and Inflationary Cosmology, Harwood Academic Publishers, (1990), New York, New York.

[19] E. Komatsu et al., Astrophys. J. Suppl. 148, 119 (2003).

[20] F. Bernardeau, L. Kofman and J. P. Uzan, Phys. Rev. D 70, 083004 (2004).

[21] G. N. Felder and L. Kofman, Phys. Rev. D 63, 103503 (2001).

[22] K. Enqvist, A. Mazumdar and M. Postma, Phys. Rev. D 67, 121303 (2003). 